\title{
Sleep-disordered breathing and stroke: chicken or egg?
}

\author{
Filip Alexiev ${ }^{1}$, Anne-Kathrin Brill ${ }^{2}$, Sebastian R. Ott ${ }^{2,3}$, Simone Duss ${ }^{1}$, Markus Schmidt ${ }^{1,4}$, Claudio L. Bassetti ${ }^{1}$ \\ ${ }^{1}$ University Sleep-Wake-Epilepsy Center (SWEZ), Department of Neurology, ${ }^{2}$ Department of Pulmonary Medicine, University Hospital (Inselspital), \\ Bern, Switzerland; ${ }^{3}$ Department of Pulmonary Medicine and Thoracic Surgery, St. Claraspital, Basel, Switzerland; ${ }^{4}$ Ohio Sleep Medicine Institute, \\ Dublin, Ohio, USA \\ Contributions: (I) Conception and design: F Alexiev, AK Brill, CL Bassetti; (II) Administrative support: None; (III) Provision of study materials: None; \\ (IV) Collection and assembly of data: F Alexiev, AK Brill; (V) Data analysis and interpretation: All authors; (VI) Manuscript writing: All authors; (VII) \\ Final approval of the manuscript: All authors. \\ Correspondence to: Filip Alexiev, MD, PhD. University Sleep-wake-Epilepsy Center (SWEZ), Department of Neurology, University Hospital \\ (Inselspital), Freiburgstrasse 18, 3010 Bern, Switzerland. Email: filip.alexiev@insel.ch.
}

\begin{abstract}
The bidirectional interaction between sleep-disordered breathing (SDB) and stroke has been the subject of many studies. On the one hand, different forms of SDB, and especially obstructive sleep apnea, increase the risk of stroke either directly or indirectly by influencing other known cardiovascular risk factors such as arterial hypertension and arrhythmias. On the other hand, stroke itself can cause either de novo appearance of SDB, aggravate a pre-existing SDB, or trigger a transition from one type of pathological SDB pattern into another. In this review, we discuss some aspects of this "chicken or egg" relationship.
\end{abstract}

Keywords: Sleep-disordered breathing (SDB); stroke; risk factors; obstructive sleep apnea (OSA); central sleep apnea (CSA)

Submitted Jun 11, 2018. Accepted for publication Nov 29, 2018.

doi: $10.21037 /$ jtd.2018.12.66

View this article at: http://dx.doi.org/10.21037/jtd.2018.12.66

\section{Introduction}

The connection between sleep-disordered breathing (SDB) and stroke was first observed in the $19^{\text {th }}$ century, but has been extensively studied only since the 1980's. Stroke remains an important cause of death and is associated with important chronic morbidity. The identification of modifiable risk factors, among them SDB, may help establish more efficient primary and secondary prevention stroke strategies. Multiple studies in the last 30 years have demonstrated the existence of a bi-directional relationship between SDB and stroke: SDB as cause (risk factor) for stroke, but also as a potential consequence of stroke. In this review, we examine this "chicken or egg" relationship (Figure 1).

\section{Sleep disordered breathing as a risk factor for stroke}

SDB is one of the most frequent causes of excessive daytime sleepiness in the general population. The prevalence of SDB as defined by an apnea + hypopnea index (AHI) greater than 5 events per hour of sleep exceeds $70 \%$, whereas the prevalence of moderate (AHI $\geq 15 / \mathrm{h}$ ) or severe (AHI $>30 / \mathrm{h}$ ) sleep apnea is $50 \%$ and $2-3 \%$ (in patients $\geq 60$ years of age over $10 \%)$, respectively $(1,2)$. Severe obstructive sleep apnea (OSA) is associated with increased overall cardiovascular mortality and plays an important role in the pathogenesis of cerebrovascular disease $(3,4)$. This has led to increased SDBscreening in patients with cerebrovascular disorders $(5,6)$.

\section{Snoring and stroke}

Studies examining the link between snoring and stroke since the 1980 `s have provided inconsistent findings. Habitual snoring of two or more nights per week was reported to be associated with a $25 \%$ higher risk of stroke (7). Although additional studies suggest that snoring could be a significant risk factor for stroke $(8,9)$, others could not find this 


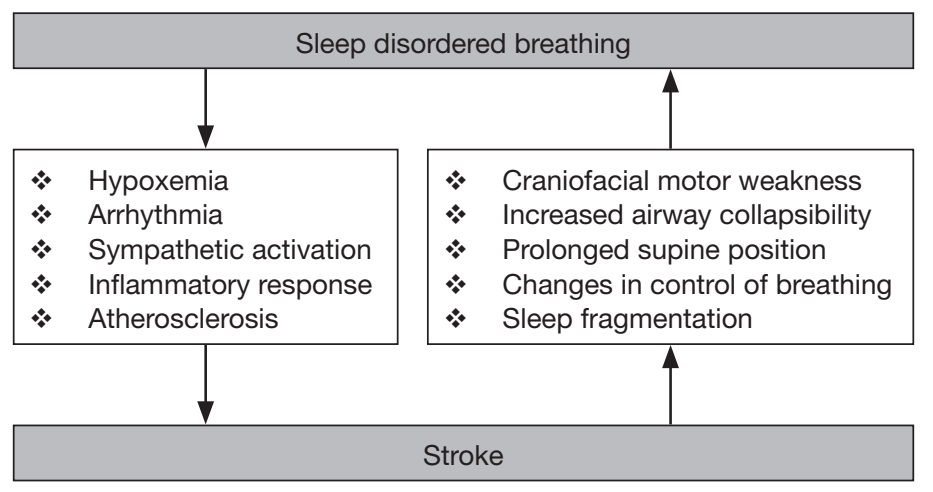

Figure 1 The bi-directional relationship between SDB and stroke. SDB, sleep-disordered breathing.

association (10). Davies et al. (11) suggest that snoring may lead to greater sleepiness during the pre-stroke period. Neau et al. (9) found that stroke risk is higher among older male patients with arterial hypertension and lifelong snoring. The link between snoring and stroke seems to be particularly strong when habitual snoring is associated with symptoms or signs suggestive of OSA such as hypersomnia, witnessed apneas and obesity (12). Snoring frequency may also adversely affect the prognosis of patients experiencing stroke (13). Despite these reported findings, habitual snoring has not significantly been associated with wake-up strokes (9).

An observational cohort study by Lee et al. (14) found that heavy snoring significantly increases the risk of carotid atherosclerosis, independently of other known risk factors, such as nocturnal hypoxia and sleep apnea severity. It has been hypothesized that snoring causes a marked vibration of the neck tissues, leading to turbulent flow through the carotid arteries, potentially contributing to atheroma formation. The Oxford Vascular Study by Mason et al. (15), however, did not find a significant association between a history of frequent snoring and the presence of carotid atheroma, degree of stenosis, or plaque type. However, snoring as a self-reported symptom can also occur in the absence of significant SDB. As a result, its reliability as an indicator of OSA-associated stroke risk remains questionable.

\section{Upper airway resistance syndrome (UARS) and stroke}

UARS and its clinical definition remains a controversial nosological entity. It is defined by the presence of respiratory effort-related arousals (RERA), such as snore arousals, that disrupt sleep without significant desaturations or associated apneas $(16,17)$. To our knowledge, there are no consistent data describing a possible connection between UARS and stroke. Compared to mild forms of OSA, patients with UARS are reported to have a worse sleep quality, as well as more fatigue and attention disturbances (18), suggesting a disrupted sleep pattern which may also be a potential risk factor for stroke (5). In addition, some authors have found UARS to increase the risk of developing treatment resistant arterial hypertension secondary to sympathetic over-activity (19), which could indirectly augment stroke risk.

\section{Obstructive sleep apnea and stroke}

OSA is characterized by either partial (hypopnea) or total (apnea) airway obstruction during sleep despite respiratory effort, resulting from a collapse of upper airway structures. OSA is very common in the general population and its association with an increased stroke and cardiovascular risk has been studied extensively. Several meta-analyses have shown that the risk of stroke is increased by a factor 2 in untreated OSA patients, especially in younger male patients. Nevertheless, it is still unclear to what extent OSA is an independent risk factor for stroke when factoring other modifiable risk factors such as arterial hypertension, ischemic heart disease, smoking or diabetes mellitus $(20,21)$.

The etiology of an increased stroke risk from SDB is suggested to be due to the acute physiological consequences and intermediary mechanisms arising from SDB such as recurrent hypoxia and sympathetic activation during sleep $(4,22,23)$. Recurrent hypoxia is accompanied by intrathoracic pressure changes, sympathetic activation and blood pressure 
swings. These acute physiological effects from recurrent breathing stoppages trigger intermediary mechanisms such as oxidative stress, endothelial dysfunction and inflammation, which lead to cardiovascular complications that include arterial hypertension, atherosclerosis, arrhythmias, hypercoagulation, heart failure and paradoxical embolism. It is well documented that OSA is an independent risk factor for cardiovascular diseases that are known to carry a major risk of stroke, such as arterial hypertension $(24,25)$. Prolonged obstructive apneas with severe oxygen desaturations (in some case over 140 s, with $\mathrm{SpO}_{2}$ nadirs below 50\%) negatively influence arterial blood pressure by causing hypoxiainduced surges with a subsequent development of recurrent symptomatic or asymptomatic strokes $(26,27)$. OSA also has a strong association with atrial fibrillation (AF), coronary artery disease, congestive heart failure, and diabetes, which are independent risk factors for stroke $(28,29)$.

Acute and immediate causes of stroke, possibly related to SDB, include long apneas with a subsequent possible rightto-left shunting that may facilitate stroke due to paradoxical embolism (30). In a retrospective, case-control, study, Lipford et al. (31) identify a strong association between OSA and cardioembolic stroke with paroxysmal AF as a probable causative factor. The negative impact of OSA has been demonstrated in another retrospective study with more than 5,000 patients where stroke was more common in patients with the comorbidity of AF and OSA (32). In a recent study focused on the relationship between poststroke OSA and stroke etiology, Poli et al. (33) also conclude that $\mathrm{AF}$ with subsequent cardioembolism is a major source of stroke based on a prospective analysis of 134 patients, showing twice the proportion of cardioembolic stroke in SDB patients (41.1\% SDB patients $v$ s. $20.5 \%$ of non-SDB patients).

\section{Central sleep apnea (CSA) and stroke}

CSA is characterized by cyclic fluctuations in respiratory drive with repetitive cessations or decreases in both airflow and ventilatory effort alternating with hyperpnea during sleep $(34,35)$. The condition can be primary (i.e., idiopathic CSA) or secondary to a medical condition, drug or substance intake, or high-altitude periodic breathing. Data on CSA as risk factor for stroke are scarce. In a crosssectional prospective study by Rupprecht et al. (36), CSA was associated with the presence of autonomic dysfunction in patients with asymptomatic stenosis of the extracranial internal carotid artery, which is an independent risk factor for stroke. In a 6-year observational study in elderly people, CSA was associated with a higher incidence of stroke (incidence of 11.28 per 1,000 person-years) (37).

\section{Obesity hypoventilation syndrome (OHS) and stroke}

The triad of obesity, daytime hypoventilation, and SDB without an alternative neuromuscular, mechanical, or metabolic cause of hypoventilation define OHS. This syndrome is a complex multi-organ system disease with a significant morbidity and mortality that should be viewed as a disease entity distinct from simple obesity and OSA $(38,39)$. To our knowledge, there are no studies exploring the association between OHS and stroke. A higher BMI seems to be a risk factor for stroke (40). Nevertheless, a Korean study by Kim et al. (41) found an obesity paradox with obese stroke patients having a decreased risk of mortality compared to overweight stroke survivors (HR of 0.83 and $95 \%$ CI of $0.74-0.92$ for BMI 27.5-30; HR of 0.77 and $95 \%$ CI of $0.63-0.93$ for BMI 30-32.5). A gender-specific difference was seen in a Japanese study with a low BMI being a risk factor for all-stroke and cerebral infarction in men, while high BMI was a risk factor for allstroke in women (42).

\section{Sleep disordered breathing as a consequence of stroke}

\section{Prevalence of SDB in stroke patients}

The prevalence of SDB in stroke survivors, or after transient ischemic attack (TIA), is high at $72 \%$ for an AHI $>5 / \mathrm{h}$ in a meta-analysis published in 2010 (43). One third of stroke survivors have an AHI $>30 / \mathrm{h}$ (43). These data recently have been confirmed in meta-analyses from our group and others (44). The most common form of SDB in stroke patients is OSA. Less common are CSA and Cheyne-Stokes breathing (CSB), the frequency of which may vary depending upon the interval of recording after stroke. A few studies have shown that in the initial days after stroke, CSB is frequent and associated with older age, stroke severity/extension, and left ventricular dysfunction (45).

Not infrequently, stroke patients present with complex SDB involving a combination of obstructive [primarily in rapid eye movement sleep (REM) sleep] and central [primarily in light non-rapid eye movement (NREM) 
sleep] breathing events. Stroke may cause (or worsen preexisting) SDB through multiple mechanisms. These include a disturbed coordination of upper airway, intercostal, and diaphragmatic muscles due to supra- or infratentorial stroke potentially favoring OSA. In patients with medullary lesions, a reduced ventilatory sensitivity to inhaled $\mathrm{CO}_{2}$, together with damage to brainstem respiratory drive centers, can predispose to apneas (46). Other factors, including hypoxemia due to aspiration or respiratory infections, reduction of voluntary chest movements on the paralyzed side, prolonged supine position, medications, and sleep fragmentation secondary to stroke complications (e.g., pain, depression) may also play a role. An interesting study of Brown et al. (47) shows that approximately $40 \%$ of ischemic stroke patients have hypoglossal nerve dysfunction that may contribute to SDB. Prolonged supine body position, which is very common in the acute stroke phase, may exacerbate positional SDB in up to $25 \%$ of stroke patients $(48,49)$. The evolution of SDB severity following stroke is less clear and has been described to be either stable in severity in group comparisons (43) or to decrease in severity during recovery in longitudinal studies (50-53).

\section{Stroke topography and SDB}

Evidence suggests that damage to specific brain structures can affect breathing patterns in both wakefulness and sleep. Several studies have failed to identify a relationship between stroke topography and type or severity of SDB (54). Other studies have found an association between both SDB severity and type with specific topographies of stroke. For example, one study suggests that the frequency of SDB is higher after brainstem stroke (55). A few observations suggest that CSA may be related to specific lesions affecting the central autonomic network $(45,56)$. Several case reports have suggested that stroke location may impact the type or severity of SDB. Faludi et al. (57) describe a case of stroke in the bilateral paramedian mesencephalon and thalamus with evolution of SDB from CSA to OSA. Focal ischemic lesions in the medulla oblongata have been shown to cause CSA with a necessity of mechanical ventilation and slow recovery. Planjar-Prvan et al. (58) present two cases of fatal CSA in patients with infarcts in the medulla oblongata at the level of the solitary tract nuclei. On the other hand, De Paolis et al. (59) describe a shift of a preexisting OSA into a severe CSA after an ischemic stroke located in the posterior limb of the left internal capsule and corona radiata.

\section{Stroke types, etiology and SDB}

Although several studies report that hemorrhagic stroke is frequently associated with $\mathrm{SDB}(60)$, one meta-analysis did not find a difference in prevalence when compared to ischemec stroke (43). Lacunar strokes were associated with greater severity of OSA with higher AHIs than cortical strokes in one study (52).

Specific stroke etiologies were also found in some but not all studies to be associated with the presence or severity of SDB. In a prospective study of 335 stroke patients, Ciccone et al. (30) identified significantly more wake-up strokes/TIAs in patients with right- to left shunts and long obstructive apneas $(>20 \mathrm{~s})$ than in patients without this association [odds ratio (OR) 1.91]. The authors concluded that this finding supports the hypothesis that long episodes of OSA can induce paradoxical embolism. Cardioembolic strokes were also seen more often in a retrospective study in stroke survivors with OSA than controls (AHI $<10 / \mathrm{h}$ ) with the majority of cardioembolic strokes in those with moderate to severe OSA (31). In the same study, AF was also more frequent in stroke patients with OSA. A prospective study with 152 patients found that a macroangiopathic etiology of stroke was significantly more common in patients with severe SDB $(\mathrm{AHI}>30 / \mathrm{h})$ than in patients with an AHI $<10 / \mathrm{h}(53)$, while Brown et al. (61) did not find an association between $\mathrm{AHI}$ and stroke subtypes.

\section{Long-term effects of SDB in stroke patients}

SDB increases mortality risk and worsens both short and long-term functional outcomes, particularly when the SDB is under- or untreated $(62,63)$. In comparison with the other stroke patients, those with SDB appear to have a higher National Institutes of Health Stroke Scale (NIHSS) and modified Rankin Scale (mRS) scores, as well as higher incidence of sleep-associated stroke onset (64). Aaronson et al. (65) demonstrate that patients with OSA and stroke in the rehabilitation phase have a lower cognitive and functional status with respect to tests of attention, executive functioning, visual perception, psychomotor ability, and intelligence. Severe OSA is associated with poor long-term functional outcomes (66).

Two systematic reviews suggest that OSA might be a 
negative predictor for all-cause mortality and recurrent vascular events following stroke or TIA $(67,68)$. In addition, the results of the meta-analysis by Birkbak et al. (67) covering ten articles with 1,203 stroke and TIA patients support a dose-response relationship between the severity of SDB and these adverse outcomes. In the second metaanalysis, OSA was associated with a pooled relative risk of 1.94 (95\% CI, 1.29-2.92) for stroke and 1.59 (95\% CI, 1.33-1.89) for all-cause mortality (68). An observational study found that CSA was not related to early death, but the study suggested that both OSA and CSA increase the longterm mortality risk in stroke patients (69).

As SDB treatment options exist, SDB after stroke is considered as a potential modifiable risk factor. A recent meta-analysis of continuous positive airway pressure (CPAP) as treatment for SDB after stroke by our group, in addition to ten randomized controlled trial (RCTs) with 564 participants, showed neurofunctional improvement with CPAP [standardized mean difference (SMD) 0.5406; 95\% CI, 0.0263-1.0548] in the combined analysis of neurofunctional scales with, however, a considerable heterogeneity across the studies (70). In one study over 5 years, the long-term cardiovascular survival was better with CPAP compared to usual care; however, there was no significant difference in the overall cardiovascular eventfree survival. In addition, the sample size of the trial was limited (71). On the other hand, it is meaningful to note that compliance with CPAP may be an important aspect with respect to the role of CPAP in cardiovascular risk reduction. Most studies consider the CPAP treatment arm as an "intention to treat" population that is considered a single or homogeneous group even if many subjects within the group have been non-compliant. If one compares only those patients who are compliant on CPAP treatment, with respect to those without CPAP, a reduction of stroke risk is then found (22). A recent randomized controlled trial of Bravata et al. (72) conclude that a better compliance to CPAP in stroke patients reduce the NIHSS score.

Table 1 summarizes the results of outcomes of some studies with positive airway pressure treatment after stroke and TIA.

\section{Conclusions}

There is a complex bidirectional relationship between
SDB and stroke. This complexity stems from the fact that SDB and stroke share common risk factors (e.g., obesity, hypertension, alcohol use, diabetes, etc.). These common risk factors may not only contribute to stroke risk in sleep apnea patients, but also potentially exacerbate functional outcomes in this patient population. Moreover, stroke can cause (or aggravate pre-existing) SDB. Although the extent, underlying mechanisms and implications of this relationship remain in parts unclear (unfortunately, interventional studies in this context are rare), current knowledge allows one to deduce the following conclusions:

(I) SDB likely increases (independently from co-shared risk factors) the risk for stroke. This association should be taken into consideration when counseling and treating patients in the context of primary and secondary stroke prevention;

(II) $\mathrm{SDB}$ is frequent in patients with stroke and is probably associated with short- and long-term detrimental effects, which can possibly be prevented or alleviated by treatment of SDB. This awareness should be promoted among neurologists and other health care professionals in the management of stroke patients. Available strategies for the detection of SDB in patients with cerebrovascular disorders/stroke should be implemented, including the use of appropriate questionnaires and portable diagnostic testing (i.e., respirographies). Currently, there are two studies in our center assessing the effects of SDB on short- and long-term outcomes in stroke/TIA patients [SAS-CARE study (73), clinicaltrials.gov NCT01097967, sleep disorders and stroke outcome study clinicaltrials.gov NCT02559739] as well as the impact of early SDB treatment with adaptive servo-ventilation on MRIevolution of stroke (eSATIS study, clinicaltrials.gov NCT02554487). More studies such as these will be needed to establish practice parameter guidelines for stroke patients with SDB;

(III) The interaction between SDB and stroke represents an area of multidisciplinary research of increasing interest, promising novel insights which may be relevant not only for the care of patients with cardiocerebrovascular disorders, but also for elucidating our understanding of brain-heart-lung interactions in health and disease. 


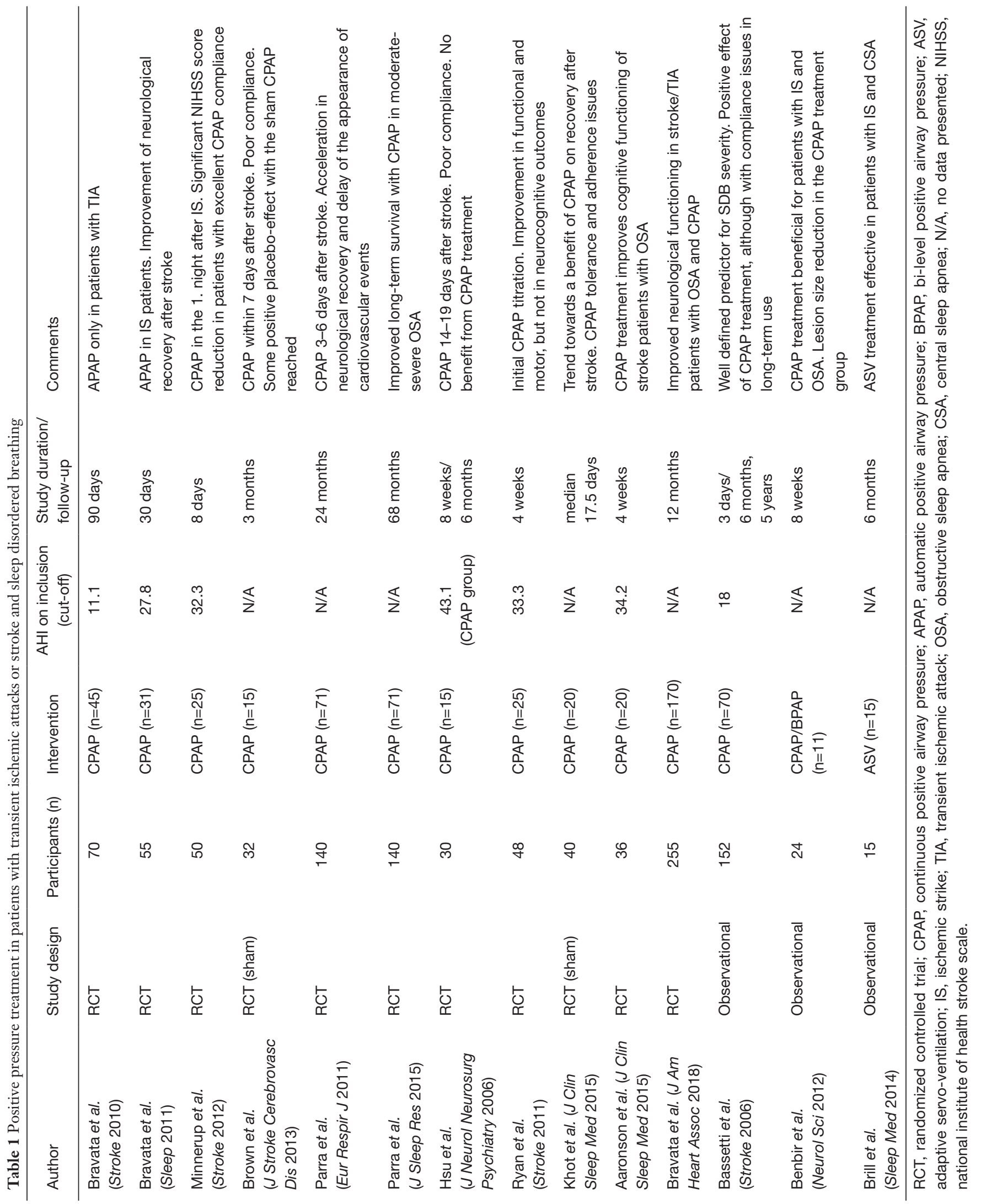




\section{Acknowledgements}

None.

\section{Footnote}

Conflicts of Interest: The authors have no conflicts of interest to declare.

\section{References}

1. Arnardottir ES, Bjornsdottir E, Olafsdottir KA, et al. Obstructive sleep apnoea in the general population: highly prevalent but minimal symptoms. Eur Respir J 2016;47:194-202.

2. Heinzer R, Vat S, Marques-Vidal P, et al. Prevalence of sleep-disordered breathing in the general population: the HypnoLaus study. Lancet Respir Med 2015;3:310-8.

3. Davis AP, Billings ME, Longstreth WT Jr, et al. Early diagnosis and treatment of obstructive sleep apnea after stroke: Are we neglecting a modifiable stroke risk factor? Neurol Clin Pract 2013;3:192-201.

4. Jonas DE, Amick HR, Feltner C, et al. Screening for Obstructive Sleep Apnea in Adults: Evidence Report and Systematic Review for the US Preventive Services Task Force. JAMA 2017;317:415-33.

5. Hermann DM, Bassetti CL. Role of sleep-disordered breathing and sleep-wake disturbances for stroke and stroke recovery. Neurology 2016;87:1407-16.

6. Kernan WN, Ovbiagele B, Black HR, et al. Guidelines for the prevention of stroke in patients with stroke and transient ischemic attack: a guideline for healthcare professionals from the American Heart Association/ American Stroke Association. Stroke 2014;45:2160-236.

7. Li M, Hou WS, Zhang XW, et al. Obstructive sleep apnea and risk of stroke: a meta-analysis of prospective studies. Int J Cardiol 2014;172:466-9.

8. Romaniak A, Stepie A. Sleep disorders as a risk factors for stroke. Neurol Neurochir Pol 2001;35:821-7.

9. Neau JP, Meurice JC, Paquereau J, et al. Habitual snoring as a risk factor for brain infarction. Acta Neurol Scand 1995;92:63-8.

10. Marshall NS, Wong KK, Cullen SR, et al. Snoring is not associated with all-cause mortality, incident cardiovascular disease, or stroke in the Busselton Health Study. Sleep 2012;35:1235-40.

11. Davies DP, Rodgers H, Walshaw D, et al. Snoring, daytime sleepiness and stroke: a case-control study of first- ever stroke. J Sleep Res 2003;12:313-8.

12. Bassetti C. Habitual snoring, sleep apnoea, and stroke prevention. J Neurol Neurosurg Psychiatry 1997;62:303.

13. Spriggs DA, French JM, Murdy JM, et al. Snoring increases the risk of stroke and adversely affects prognosis. Q J Med 1992;83:555-62.

14. Lee SA, Amis TC, Byth K, et al. Heavy snoring as a cause of carotid artery atherosclerosis. Sleep 2008;31:1207-13.

15. Mason RH, Mehta Z, Fonseca AC, et al. Snoring and severity of symptomatic and asymptomatic carotid stenosis: a population-based study. Sleep 2012;35:1147-51.

16. M'saad S, Yangui I, Feki W, et al. The syndrome of increased upper airways resistance: What are the clinical features and diagnostic procedures? Rev Mal Respir 2015;32:1002-15.

17. Pépin JL, Guillot M, Tamisier R, et al. The upper airway resistance syndrome. Respiration 2012;83:559-66.

18. de Godoy LB, Luz GP, Palombini LO, et al. Upper Airway Resistance Syndrome Patients Have Worse Sleep Quality Compared to Mild Obstructive Sleep Apnea. PLoS One 2016;11:e0156244.

19. Asker M, Asker S, Kucuk U, et al. An overlooked cause of resistant hypertension: upper airway resistance syndrome preliminary results. Clinics (Sao Paulo) 2014;69:731-4.

20. Kendzerska T, Mollayeva T, Gershon AS, et al. Untreated obstructive sleep apnea and the risk for serious long-term adverse outcomes: a systematic review. Sleep Med Rev 2014;18:49-59.

21. Loke YK, Brown JW, Kwok CS, et al. Association of obstructive sleep apnea with risk of serious cardiovascular events: a systematic review and meta-analysis. Circ Cardiovasc Qual Outcomes 2012;5:720-8.

22. Javaheri S, Barbe F, Campos-Rodriguez F, et al. Sleep Apnea: Types, Mechanisms, and Clinical Cardiovascular Consequences. J Am Coll Cardiol 2017;69:841-58.

23. Shamsuzzaman AS, Gersh BJ, Somers VK. Obstructive sleep apnea: implications for cardiac and vascular disease. JAMA 2003;290:1906-14.

24. Marin JM, Agusti A, Villar I, et al. Association between treated and untreated obstructive sleep apnea and risk of hypertension. JAMA 2012;307:2169-76.

25. Barbé F, Durán-Cantolla J, Sánchez-de-la-Torre $M$, et al. Effect of continuous positive airway pressure on the incidence of hypertension and cardiovascular events in nonsleepy patients with obstructive sleep apnea: a randomized controlled trial. JAMA 2012;307:2161-8.

26. Yoshida T, Kuwabara M, Hoshide S, et al. Recurrence of stroke caused by nocturnal hypoxia-induced blood pressure 
surge in a young adult male with severe obstructive sleep apnea syndrome. J Am Soc Hypertens 2016;10:201-4.

27. Sacco S, Peverini F, Bastianello S, et al. Severe oxygen desaturation in obstructive sleep-apnea syndrome as a possible cause of ischemic stroke. Eur Neurol 2005;53:86-7.

28. Rich J, Raviv A, Raviv N, et al. All-cause mortality and obstructive sleep apnea severity revisited. Otolaryngol Head Neck Surg 2012;147:583-7.

29. Capampangan DJ, Wellik KE, Parish JM, et al. Is obstructive sleep apnea an independent risk factor for stroke? A critically appraised topic. Neurologist 2010;16:269-73.

30. Ciccone A, Proserpio P, Roccatagliata DV, et al. Wake-up stroke and TIA due to paradoxical embolism during long obstructive sleep apnoeas: a cross-sectional study. Thorax 2013;68:97-104.

31. Lipford MC, Flemming KD, Calvin AD, et al. Associations between Cardioembolic Stroke and Obstructive Sleep Apnea. Sleep 2015;38:1699-705.

32. Yaranov DM, Smyrlis A, Usatii N, et al. Effect of obstructive sleep apnea on frequency of stroke in patients with atrial fibrillation. Am J Cardiol 2015;115:461-5.

33. Poli M, Philip P, Taillard J, et al. Atrial fibrillation is a major cause of stroke in apneic patients: a prospective study. Sleep Med 2017;30:251-4.

34. Randerath W, Verbraecken J, Andreas S, et al. Definition, discrimination, diagnosis and treatment of central breathing disturbances during sleep. Eur Respir J 2017;49.

35. American Academy of Sleep Medicine. International Classification of Sleep Disorders. 3rd edition. Darien, IL: American Academy of Sleep Medicine, 2014.

36. Rupprecht S, Hoyer D, Hagemann G, et al. Central sleep apnea indicates autonomic dysfunction in asymptomatic carotid stenosis: a potential marker of cerebrovascular and cardiovascular risk. Sleep 2010;33:327-33.

37. Muñoz R, Durán-Cantolla J, Martinez-Vila E, et al. Central sleep apnea is associated with increased risk of ischemic stroke in the elderly. Acta Neurol Scand 2012;126:183-8.

38. Chau EH, Lam D, Wong J, et al. Obesity hypoventilation syndrome: a review of epidemiology, pathophysiology, and perioperative considerations. Anesthesiology 2012;117:188-205.

39. Pierce AM, Brown LK. Obesity hypoventilation syndrome: current theories of pathogenesis. Curr Opin Pulm Med 2015;21:557-62.

40. O'Donnell MJ, Chin SL, Rangarajan S, et al. Global and regional effects of potentially modifiable risk factors associated with acute stroke in 32 countries (INTERSTROKE): a case-control study. Lancet 2016;388:761-75.

41. Kim BJ, Lee SH, Jung KH, et al. Dynamics of obesity paradox after stroke, related to time from onset, age, and causes of death. Neurology 2012;79:856-63.

42. Kawate N, Kayaba K, Hara M, et al. Body mass index and stroke incidence in Japanese community residents: The Jichi Medical School (JMS) Cohort Study. J Epidemiol 2017;27:325-30.

43. Johnson KG, Johnson DC. Frequency of sleep apnea in stroke and TIA patients: a meta-analysis. J Clin Sleep Med 2010;6:131-7.

44. Dong R, Dong Z, Liu H, et al. Prevalence, Risk Factors, Outcomes, and Treatment of Obstructive Sleep Apnea in Patients with Cerebrovascular Disease: A Systematic Review. J Stroke Cerebrovasc Dis 2018;27:1471-80.

45. Siccoli MM, Valko PO, Hermann DM, et al. Central periodic breathing during sleep in 74 patients with acute ischemic stroke - neurogenic and cardiogenic factors. J Neurol 2008;255:1687-92.

46. Morrell MJ, Heywood P, Moosavi SH, et al. Unilateral focal lesions in the rostrolateral medulla influence chemosensitivity and breathing measured during wakefulness, sleep, and exercise. J Neurol Neurosurg Psychiatry 1999;67:637-45.

47. Brown DL, Chervin RD, Wolfe J, et al. Hypoglossal nerve dysfunction and sleep-disordered breathing after stroke. Neurology 2014;82:1149-52.

48. Camilo MR, Fernandes RM, Sander HH, et al. Supine sleep and positional sleep apnea after acute ischemic stroke and intracerebral hemorrhage. Clinics (Sao Paulo) 2012;67:1357-60.

49. Brown DL, Lisabeth LD, Zupancic MJ, et al. High prevalence of supine sleep in ischemic stroke patients. Stroke 2008;39:2511-4.

50. Bravata DM, Concato J, Fried T, et al. Continuous positive airway pressure: evaluation of a novel therapy for patients with acute ischemic stroke. Sleep 2011;34:1271-7.

51. Parra O, Arboix A, Bechich S, et al. Time course of sleep-related breathing disorders in first-ever stroke or transient ischemic attack. Am J Respir Crit Care Med 2000;161:375-80.

52. Harbison J, Ford GA, James OF, et al. Sleep-disordered breathing following acute stroke. QJM 2002;95:741-7.

53. Bassetti CL, Milanova M, Gugger M. Sleep-disordered breathing and acute ischemic stroke: diagnosis, risk factors, 
treatment, evolution, and long-term clinical outcome. Stroke 2006;37:967-72.

54. Fisse AL, Kemmling A, Teuber A, et al. The Association of Lesion Location and Sleep Related Breathing Disorder in Patients with Acute Ischemic Stroke. PLoS One 2017;12:e0171243.

55. Brown DL, McDermott M, Mowla A, et al. Brainstem infarction and sleep-disordered breathing in the BASIC sleep apnea study. Sleep Med 2014;15:887-91.

56. Hermann DM, Bassetti CL. Sleep Apnea and Other SleepWake Disorders in Stroke. Curr Treat Options Neurol 2003;5:241-9.

57. Faludi B, Tóth M, Pusch G, et al. Dynamic changes in sleep-related breathing abnormalities in bilateral paramedian mesencephalon and thalamus stroke: a followup case study. Sleep Breath 2016;20:237-42.

58. Planjar-Prvan M, Krmpotić P, Jergović I, et al. Central sleep apnea (Ondine's curse syndrome) in medullary infarction. Acta Med Croatica 2010;64:297-301.

59. De Paolis F, Colizzi E, Milioli G, et al. Acute shift of a case of moderate obstructive sleep apnea syndrome towards one of severe central sleep apnea syndrome after an ischemic stroke. Sleep Med 2012;13:763-6.

60. Pontes-Neto OM, Fernandes RM, Sander HH, et al. Obstructive sleep apnea is frequent in patients with hypertensive intracerebral hemorrhage and is related to perihematoma edema. Cerebrovasc Dis 2010;29:36-42.

61. Brown DL, Mowla A, McDermott M, et al. Ischemic stroke subtype and presence of sleep-disordered breathing: the BASIC sleep apnea study. J Stroke Cerebrovasc Dis 2015;24:388-93.

62. Young T, Finn L, Peppard PE, et al. Sleep disordered breathing and mortality: eighteen-year follow-up of the Wisconsin sleep cohort. Sleep 2008;31:1071-8.

63. Good DC, Henkle JQ, Gelber D, et al. Sleep-disordered breathing and poor functional outcome after stroke. Stroke 1996;27:252-9.

64. Ahn SH, Kim JH, Kim DU, et al. Interaction between

Cite this article as: Alexiev F, Brill AK, Ott SR, Duss S, Schmidt M, Bassetti CL. Sleep-disordered breathing and stroke: chicken or egg? J Thorac Dis 2018;10(Suppl 34):S4244-S4252. doi: 10.21037/jtd.2018.12.66
Sleep-Disordered Breathing and Acute Ischemic Stroke. J Clin Neurol 2013;9:9-13.

65. Aaronson JA, van Bennekom CA, Hofman WF, et al. Obstructive Sleep Apnea is Related to Impaired Cognitive and Functional Status after Stroke. Sleep 2015;38:1431-7.

66. Camilo MR, Schnitman SV, Sander HH, et al. Sleepdisordered breathing among acute ischemic stroke patients in Brazil. Sleep Med 2016;19:8-12.

67. Birkbak J, Clark AJ, Rod NH. The effect of sleep disordered breathing on the outcome of stroke and transient ischemic attack: a systematic review. J Clin Sleep Med 2014;10:103-8.

68. Xie W, Zheng F, Song X. Obstructive sleep apnea and serious adverse outcomes in patients with cardiovascular or cerebrovascular disease: a PRISMA-compliant systematic review and meta-analysis. Medicine (Baltimore) 2014;93:e336.

69. Sahlin C, Sandberg O, Gustafson Y, et al. Obstructive sleep apnea is a risk factor for death in patients with stroke: a 10-year follow-up. Arch Intern Med 2008;168:297-301.

70. Brill AK, Horvath T, Seiler A, et al. CPAP as treatment of sleep apnea after stroke: A meta-analysis of randomized trials. Neurology 2018;90:e1222-30.

71. Parra O, Sánchez-Armengol Á, Capote F, et al. Efficacy of continuous positive airway pressure treatment on 5-year survival in patients with ischaemic stroke and obstructive sleep apnea: a randomized controlled trial. J Sleep Res 2015;24:47-53.

72. Bravata DM, Sico J, Vaz Fragoso CA, et al. Diagnosing and Treating Sleep Apnea in Patients With Acute Cerebrovascular Disease. J Am Heart Assoc 2018; 7:e008841.

73. Cereda CW, Petrini L, Azzola A, et al. Sleep-disordered breathing in acute ischemic stroke and transient ischemic attack: effects on short- and long-term outcome and efficacy of treatment with continuous positive airways pressure--rationale and design of the SAS CARE study. Int J Stroke 2012;7:597-603. 